\title{
How does substrate hydrophobicity affect the morphological features of reconstituted wax films and their interactions with nonionic surfactant and pesticide?
} DOI:

10.1016/j.jcis.2020.04.043

\section{Document Version \\ Accepted author manuscript}

Link to publication record in Manchester Research Explorer

Citation for published version (APA):

Hu, X., Pambou, E., Gong, H., Liao, M., Liu, H., Hollowell, P., \& Lu, J. R. (2020). How does substrate hydrophobicity affect the morphological features of reconstituted wax films and their interactions with nonionic surfactant and pesticide? Journal of Colloid and Interface Science, 575, 245-253.

https://doi.org/10.1016/j.jcis.2020.04.043

Published in:

Journal of Colloid and Interface Science

\section{Citing this paper}

Please note that where the full-text provided on Manchester Research Explorer is the Author Accepted Manuscript or Proof version this may differ from the final Published version. If citing, it is advised that you check and use the publisher's definitive version.

\section{General rights}

Copyright and moral rights for the publications made accessible in the Research Explorer are retained by the authors and/or other copyright owners and it is a condition of accessing publications that users recognise and abide by the legal requirements associated with these rights.

\section{Takedown policy}

If you believe that this document breaches copyright please refer to the University of Manchester's Takedown Procedures [http://man.ac.uk/04Y6Bo] or contact uml.scholarlycommunications@manchester.ac.uk providing relevant details, so we can investigate your claim.

\section{OPEN ACCESS}




\section{How Does Substrate Hydrophobicity Affect the Morphological Features of Reconstituted Wax Films and Their Interactions with Nonionic Surfactant and Pesticide?}

Xuzhi Hu ${ }^{a}$, Elias Pambou ${ }^{a}$, Haoning Gong ${ }^{a}$, Mingrui Liao ${ }^{a}$, Peter Hollowell ${ }^{a}$, Huayang Liu ${ }^{a}$, Weimiao Wang ${ }^{b}$, Carlo Bawn ${ }^{c}$, Jos Cooper ${ }^{d}$, Mario Campana ${ }^{d}$,Kun Ma , Peixun Li ${ }^{d}$, John R.P. Webster ${ }^{d}$,Faheem Padia ${ }^{e}$,Gordon Bell , Jian R. Lu ${ }^{a^{*}}$

a Biological Physics Group, School of Physics and Astronomy, University of Manchester, Oxford Road, Manchester, M13 9PL, UK.

${ }^{\mathrm{b}}$ Department of Materials and National Graphene Institute, University of Manchester, Oxford Road, Manchester, M13 9PL, UK.

${ }^{c}$ School of Chemistry, University of Manchester, Oxford Road, Manchester, M13 9PL, UK.

${ }^{\mathrm{d}}$ STFC ISIS Facility, Rutherford Appleton Laboratory, Didcot, OX11 0QX, UK.

e Syngenta, Jealott's Hill International Research Centre, Bracknell, Berkshire, RG42 6EY, UK.

* E-mail: j.lu@ manchester.ac.uk. Tel: (+44)-161-200-3926.

Keywords: Wheat, Triticum, plant waxes, deuterated wax, neutron reflection, spectroscopic ellipsometry, nonionic surfactant, pesticide 
TOC:
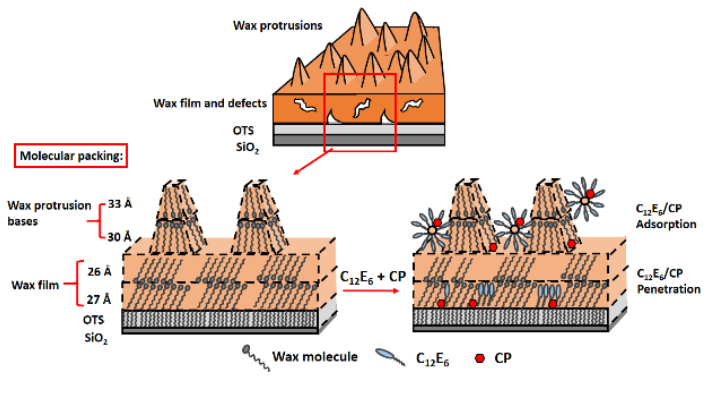


\section{Abstract \\ Hypothesis}

Surfactants are widely used in agri-sprays to improve pesticide efficiency, but the mechanism underlying their interactions with the surface wax film on plants remains poorly understood. To facilitate physical characterisations, we have reconstituted wheat cuticular wax films onto an optically flat silicon substrate with and without octadecyltrimethoxysilane modification to control surface hydrophobicity.

\section{Experiments}

Imaging techniques including scanning electron microscopy (SEM) unravelled morphological features of the reconstituted wax films similar to those on leaves, showing little impact from the different substrates used. Neutron reflection (NR) established that reconstituted wax films were comprised of an underlying wax film decorated with top surface wax protrusions, a common feature irrespective of substrate hydrophobicity and highly consistent with what was observed from natural wax films. NR measurements, with the help of isotopic H/D substitutions to modify the scattering contributions of the wax and solvent, revealed different wax regimes within the wax films, illustrating the impact of surface hydrophilicity on the nanostructures within the wax films.

\section{Findings}

It was observed from both spectroscopic ellipsometry and NR measurements that wax films formed on the hydrophobic substrate were more robust and durable against attack by nonionic surfactant $\mathrm{C}_{12} \mathrm{E}_{6}$ solubilised with pesticide Cyprodinil (CP) than films coated on the bare hydrophilic silica. Thus, the former could be a more feasible model for studying the wax-surfactant-pesticide interactions. 


\section{Introduction}

Pesticides are essential in modern agriculture for protecting crops from various pest infestations and ensuring high yields and high quality of the harvests $[1,2]$. Commercial pesticide formulations normally rely on adjuvants such as nonionic surfactants $[3,4]$ to improve pesticide solubilisation [5], mobility [6], bouncing reduction [7, 8] and surface coverage [9]. Upon spraying, the mixture of adjuvants and pesticides first contacts the plant outer surface which is fully covered by a wax film [10]. The spray forms a thin watery film of the formulation or an array of its droplets on the wax film surface. As water evaporates, the concentrations increase, driving adjuvants and pesticides into the leaves and stems. $[11,12]$ It is crucial to examine the interactions between wax film, surfactant and pesticide on the plant surface, as this process is vital to understanding how to protect crops more effectively whilst minimising the use of pesticides and adjuvants.

Surfactants readily adsorb at different interfaces and their interaction with wax film underlines the uptake of pesticide molecules into leaves. At the same time, surfactants form micellar aggregate in the aqueous solution above their critical aggregation concentrations (CACs). It is well expected that surfactant micellar properties affect pesticide loading and subsequent wax solubilisation. Although agrichemical industry has decades of practices in the field little has been reported in the open literature describing these interactions. Because most pesticides are rather hydrophobic with different molecular structures, the exact amount and location of a pesticide into the micelles of a nonionic surfactant are affected by not only their own molecular structures but also environment conditions. As a result, pesticide solubilisation generally alters micellar phase boundaries by decreasing the cloud point and changes in micellar shape. Our recent study has revealed the structural changes of the nonionic surfactant micelles including elongation of the overall shape and shrinkage and dehydration of the shell, resulting in increased micellar hydrophobicity [13]. Dissolution of wheat waxes into the pesticide saturated micelles can trigger the release of the pesticides from the micelles due to the re-equilibration of the micellar system [14]. These recent studies have thus laid a useful basis for us to further explore how surfactants and pesticides interact with wax films. These studies together will provide us a picture of molecular processes underlying the transport of pesticides across the waxy interface, loss of wax molecules from plant surface and the role of nonionic surfactants.

Despite extensive in vivo studies involving real plants [10, 15-20], the molecular processes underlying these interactions remain largely unexplored as most surface techniques cannot be 
directly applied to the leaf surface. To overcome this limitation, a novel strategy using reconstituted wax films was employed to study wax behaviour when exposed to pesticides and adjuvants [21]. We have demonstrated a reconstituting method by first extracting waxes from wheat leaves and then forming wax films onto silicon oxide surface by spin coating. The reconstituted wheat wax films share similar structural features with natural wheat waxes [22]. When employing neutron reflection (NR) combined with deuterium labelling to either surfactant or water, we have shown that nonionic surfactants can adsorb onto and penetrate into the reconstituted wax film. It was also observed that above the CAC of the surfactant, a certain amount of wax can be removed by the micelles. However, as the coated wax film to hydrophilic silicon oxide surface was not stable, it was largely disrupted by the nonionic surfactant at high concentrations [23]. This inhibits the further investigation on tracing the surfactant and pesticide from the binary model formulation mimicking the formulated commercial products containing pesticides. Thus, a stronger adhesion between the wax film and the substrate must be developed.

In this work wax films were spin-coated onto a hydrophobic self-assembled monolayer (SAM) composed of octadecyltrimethoxysilane (OTS) on an optically flat silicon oxide surface. It was hoped the OTS modified substrate surface would offer strong adhesion to the wax film and better stability. To enhance interfacial structural resolution, deuterated wax film was used. In NR experiments, H/D substitution changes the scattering length density (SLD) of the interface which enables measurements to be performed in different contrasts [24]. To enhance the structural sensitivity of NR and trace the surfactant and pesticide molecule, it is beneficial to deuterate surfactants and vary the isotopic contrasts of the solvent in addition to the introduction of the deuterated waxes. Building from our previous studies [13, 14, 22, 23], we again selected Cyprodinil (CP, 4-cyclopropyl-6-methyl-N-phenylpyrimidin-2-amine) as a model fungicide and saturated it into the micelles of nonionic surfactant hexaethylene glycol monododecyl ether $\left(\mathrm{C}_{12} \mathrm{E}_{6}\right)$. Wheat waxes were chosen as their average molecular structure has been well characterised [14]. Deuterated waxes were extracted from wheat leaves grown in $\mathrm{D}_{2} \mathrm{O}$. The structures of Cyprodinil, $\mathrm{C}_{12} \mathrm{E}_{6}$, and the representative wheat wax molecule are shown in Figure 1.

The structural features of wax films formed on both silica and OTS treated surface have been characterised to investigate the influences of substrate surface hydrophilicity on the structures of the reconstituted wax films, followed by a further study into their interaction with $\mathrm{C}_{12} \mathrm{E}_{6}$ and CP. 
(a) Cyprodinil (CP):<smiles>Cc1cc(C2CC2)nc(Nc2ccccc2)n1</smiles>

(b) $\mathrm{C}_{12} \mathrm{E}_{6}$ :

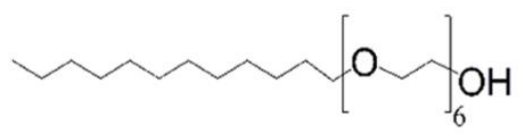

(c) Wheat wax:

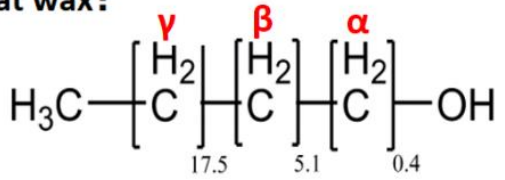

Figure 1: Molecular structures of (a) $\mathrm{CP}$, (b) $\mathrm{C}_{12} \mathrm{E}_{6}$ and (c) wheat wax with $\alpha, \beta$ and $\gamma$ methylene groups marked for further interpretation

In addition to NR, proton nuclear magnetic resonance $\left({ }^{1} \mathrm{H}-\mathrm{NMR}\right)$ was applied to examining the extent of deuteration on deuterated wax molecules. Scanning electron microscopy (SEM) and NR were used to characterise the reconstituted wax film morphologies and structures. Spectroscopic ellipsometry (SE) and NR were employed to study how nonionic surfactant and pesticide molecules interacted with the wax films. This study thereby not only helps understand the interactions between waxes, pesticides and surfactants at plant surface but also establish the basis of future pesticide formulation with smart pesticide release, which is an important application of colloid and interface science.

\section{Experimental methods}

\subsection{Materials and $h / d-w a x$ preparations}

The Triticum aestivum L. (winter wheat) seeds were supplied by Syngenta Jealott's Hill International Research Centre. $\mathrm{D}_{2} \mathrm{O}$ (99.9\% atom D\%, Sigma Aldrich Co. Ltd), octadecyltrimethoxysilane denoted as OTS (90\%, Sigma Aldrich Co. Ltd), hexaethylene glycol monododecyl ether denoted as $\mathrm{hC}_{12} \mathrm{hE}_{6}(99 \%$, Sigma Aldrich Co. Ltd) and Cyprodinil (CP) ( $\geq 95 \%$, Sigma Aldrich Co. Ltd) were used without any further purification. The tail deuterated surfactant denoted as $\mathrm{dC}_{12} \mathrm{hE}_{6}$ (deuteration: 98\%) was synthesised by the ISIS Deuteration Laboratory, Rutherford Appleton Laboratory, STFC.

Nonionic surfactants were dissolved in $\mathrm{D}_{2} \mathrm{O}$ or mixture of $\mathrm{D}_{2} \mathrm{O}$ and $\mathrm{H}_{2} \mathrm{O}$ at a concentration of 10 times its critical micellar concentration (CMC) $(0.067 \mathrm{mM})$ [25] and CP was then solubilised into the surfactants at $20^{\circ} \mathrm{C}$ using our previous method [13].

The wheat seeds were planted in pots (diameter: $10 \mathrm{~cm}$; depth: $9 \mathrm{~cm})(20$ seeds per pot) and fed with water for 3 days at $20{ }^{\circ} \mathrm{C}$ until the wheat was about $1 \mathrm{~cm}$ high. Half of the pots were transferred into a mini greenhouse. The mini green house was pre-saturated with $\mathrm{D}_{2} \mathrm{O}$ by 
mounting a beaker of $200 \mathrm{ml}$ of $\mathrm{D}_{2} \mathrm{O}$ inside overnight. The wheats in the greenhouse were watered with a mixture of $50 \% \mathrm{D}_{2} \mathrm{O}$ for 3 days and then watered with $\mathrm{D}_{2} \mathrm{O}$ for the remaining fortnight whilst the other half remained in $\mathrm{H}_{2} \mathrm{O}$ feeding. The volume of watering was 50 $\mathrm{ml} / \mathrm{day}$.

The three-weeks-old wheat leaves were collected and submerged in chloroform for $10 \mathrm{~min}$ followed by filtration. After filtration the solvent was completely evaporated using a rotary evaporator. The extracted deuterated and protonated waxes, denoted as d-wax and h-wax, respectively, were re-dissolved in chloroform at a concentration of $0.05 \% \mathrm{w} / \mathrm{w}$ for storage and reconstituted wax film fabrication.

The extent of deuteration for the d-wax was measured by ${ }^{1} \mathrm{H}-\mathrm{NMR}$ (B400 Bruker Avance III $400 \mathrm{MHz}$ ). The dry h/d-waxes were dissolved in d-chloroform (99.9\% atom D\%, Sigma Aldrich Co. Ltd) at a concentration of $0.1 \% \mathrm{w} / \mathrm{w}$ and benzyl chloride (>99\%, Sigma Aldrich Co. Ltd) was added as an internal reference at a concentration of $0.16 \% \mathrm{w} / \mathrm{w}$. The extent of deuteration of the individual functional groups in the wax and the method to determine said deuteration levels are given in the Supporting Information.

\subsection{Surface treatment and wax film coating}

The surface treatment was improved from our previous methods [26, 27]. Before treatment, the silicon wafers/blocks were immersed in a preheated piranha solution $\left(\mathrm{H}_{2} \mathrm{SO}_{4}: \mathrm{H}_{2} \mathrm{O}_{2}\right.$ ratio of 9:1 at $90{ }^{\circ} \mathrm{C}$ for $1 \mathrm{~min}$ ), rinsed repeatedly with plenty of de-ionised water (resistivity at 18 $\mathrm{M} \Omega \mathrm{cm}$, Purelab UHQ, Vivendi Water Systems Ltd.) and dried with nitrogen gas. The wafers/blocks were then immersed in OTS at a concentration of $100 \mathrm{mM}$ in chloroform overnight. The wafers/blocks were then rinsed with ethanol and annealed in vacuum at $150{ }^{\circ} \mathrm{C}$ for $8 \mathrm{hr}$.

The $0.05 \%$ w/w wax chloroform solution was deposited onto wafers/blocks (Silicon oxide or OTS surfaces) and spin-coated (Laurell Technologies, MODEL WS-650MZ-23NPP, prehorizontalised) at 2000 RPM for 10s immediately after deposition.

For SEM and spectroscopic ellipsometry (SE) studies, the wafers utilised were cut in a size of $1.2 \mathrm{~cm} \times 1.2 \mathrm{~cm}$ and $50 \mu \mathrm{l}$ wax solution was deposited on the surface. For neutron reflectivity (NR) measurements, the blocks with dimensions of $8 \mathrm{~cm}$ (length) $\times 5 \mathrm{~cm}$ (width) $\times 1 \mathrm{~cm}$ (height) were used and $1.5 \mathrm{ml}$ solution was deposited on the large polished surface for the spin-coating procedure to commence. 


\subsection{SEM}

The SEM studies were carried out on a Hitachi SU8220. The excised leaf pieces were mounted on a specimen stub and the temperature was maintained at $-60{ }^{\circ} \mathrm{C}$. The sample was scanned at a voltage of $2.0 \mathrm{kV}$ and the working distance was maintained as 7-8.2 $\mathrm{mm}$.

\subsection{Spectroscopic ellipsometry (SE)}

SE measurements were performed in a specific liquid cell with a fixed incident angle at $70^{\circ}$ at the solid/liquid interface using a Woollam spectroscopic ellipsometer (J.A. Woollam Co. Inc). The dynamic changes of reconstituted wax films exposed to $\mathrm{C}_{12} \mathrm{E}_{6}$ and $\mathrm{CP}$ were measured to characterise the extent of surfactant and pesticide adsorption and wax removal. The reconstituted wax films coated on OTS treated wafers were set in the liquid cell and fixed in place by a sample holder. Prior to $\mathrm{C}_{12} \mathrm{E}_{6}$ and $\mathrm{CP}$ injections, the films were rinsed with plenty of de-ionised water until stabilization. Then the films were exposed to $\mathrm{C}_{12} \mathrm{E}_{6}$ at $0.3,1$, 10 and $20 \mathrm{CMC}, \mathrm{C}_{12} \mathrm{E}_{6}$ solubilised with $\mathrm{CP}$ at $10 \mathrm{CMC}$ and $\mathrm{C}_{12} \mathrm{E}_{6}$ at $10 \mathrm{CMC}$ for 10 min each. Between each exposure ample amounts of de-ionised water were washed over the surface to remove any residue. The principle of the ellipsometric technique in measuring the mass change of the film is described in the section A of the Supporting Information.

\subsection{Neutron reflectivity (NR)}

NR studies were performed on SURF in TS1 and OFFSPEC in TS2 at the ISIS Neutron Faculty, Rutherford Appleton Laboratory. The Q-range was set from 0.012 to $0.4 \AA^{-1}$ and the neutron beam illuminating region was about $3-4 \mathrm{~cm}^{2}$ defined by the slits setting. Each reflectivity profile consisted of measurements at 3 incident angles of $0.35^{\circ}, 0.7^{\circ}$ and $1.5^{\circ}$. A list of all sample SLDs involved is given in Table 1. Characterisation of the native silicon oxide film and the self-assembled OTS surface layer was carried out at the very beginning of the experiments. The wax films were then spin coated onto the $\mathrm{SiO}_{2}$ and OTS treated blocks.

\begin{tabular}{lccccccccccc}
\hline Materials & h-wax & d-wax & $\mathbf{d C}_{12}$ & $\mathbf{h E}_{6}$ & $\mathbf{h C}_{12}$ & $\mathbf{C P}$ & $\mathbf{O T S}$ & $\mathbf{D}_{2} \mathbf{O}$ & $\mathbf{H}_{2} \mathbf{O}$ & $\mathbf{S i}$ & $\mathbf{S i O}_{2}$ \\
\hline SLD $/ \times 10^{-6}$ & -0.29 & 2.2 & 6.89 & 0.63 & -0.46 & 2.1 & -0.15 & 6.35 & -0.56 & 2.07 & 3.47 \\
$\AA^{-2}$ & & & & & & & & & &
\end{tabular}

Table 1: The SLD values for all the samples used in this experiment. The SLD values, except d-wax, were taken from previous studies [13, 14, 22, 23].

Characterisations of wax films on silica and OTS surface were carried out in 4 contrasts: hwax in $\mathrm{D}_{2} \mathrm{O}$; h-wax in contrast matching to d-wax (CMd-wax, SLD $\left.=2.2 \times 10^{-6} \AA^{-2}\right)$; d-wax in $\mathrm{D}_{2} \mathrm{O}$ and d-wax in contrast matching to h-wax $\left(\mathrm{CMh}-\mathrm{wax}, \mathrm{SLD}=-0.29 \times 10^{-6} \AA^{-2}\right)$. Similar to SE measurements, the wax films were first rinsed with $\mathrm{C}_{12} \mathrm{E}_{6}$ and then $\mathrm{C}_{12} \mathrm{E}_{6}$ solubilised with 
$\mathrm{CP}$ at $10 \mathrm{CMC}$ with plenty of de-ionised water repeatedly rinsed in between sample injections. The measurements of wax- $\mathrm{C}_{12} \mathrm{E}_{6}$ interactions were performed on wax films coated on the OTS surface in 5 contrasts: h-wax with $\mathrm{hC}_{12} \mathrm{hE}_{6}$ in $\mathrm{D}_{2} \mathrm{O}$, h-wax with $\mathrm{dC}_{12} \mathrm{hE}_{6}$ in $\mathrm{D}_{2} \mathrm{O}$, h-wax with $\mathrm{dC}_{12} \mathrm{hE}_{6}$ in CMh-wax, d-wax with $\mathrm{hC}_{12} \mathrm{hE}_{6}$ in $\mathrm{D}_{2} \mathrm{O}$ and d-wax with $\mathrm{hC}_{12} \mathrm{hE}_{6}$ in CMd-wax. As the SLD for $\mathrm{d}$-wax is close to the SLD of $\mathrm{CP}$, the measurements on wax- $\mathrm{C}_{12} \mathrm{E}_{6}-\mathrm{CP}$ interactions were performed only on h-wax films in 4 contrasts: h-wax with $\mathrm{hC}_{12} \mathrm{hE}_{6}$ and $\mathrm{CP}$ in $\mathrm{D}_{2} \mathrm{O}$, h-wax with $\mathrm{dC}_{12} \mathrm{hE}_{6}$ and $\mathrm{CP}$ in $\mathrm{D}_{2} \mathrm{O}$, h-wax with $\mathrm{dC}_{12} \mathrm{hE}_{6}$ and $\mathrm{CP}$ in CMh-wax and hwax with $\mathrm{hC}_{12} \mathrm{hE}_{6}$ and $\mathrm{CP}$ in $\mathrm{CMh}-$ wax.

The reflectivity data were analysed using Motofit software [28] and a 4-layer model for wax characterisation and a 7-layer model for wax-surfactant and wax-surfactant-CP interactions were established with the detailed NR principles and fitting model included in Section B of the Supporting Information.

\section{Results and Discussion}

\subsection{Wax deuteration}

At the early stage of this work it was found that the wheat seeds failed to germinate from direct $\mathrm{D}_{2} \mathrm{O}$ watering. We then started watering with $\mathrm{H}_{2} \mathrm{O}$ for the first 3 days. After germination and when the plants were strong enough to tolerate $\mathrm{D}_{2} \mathrm{O}$, they were exposed to the gradual increase of the concentration of $\mathrm{D}_{2} \mathrm{O}$ by watering with $50 \% \mathrm{D}_{2} \mathrm{O}$ for the second 3 days and then pure $\mathrm{D}_{2} \mathrm{O}$ in the remaining time. After 3 weeks of watering, waxes were harvested and the extent of deuteration was measured by ${ }^{1} \mathrm{H}-\mathrm{NMR}$. As explained previously, the main wax components were primary alcohols with the average molecular structure already characterised in our previous work [14]. It was however found that the level of deuterium was not evenly distributed over different functional groups: $35.8 \%$ for the methyl group, $37.8 \%$ for the $\alpha$-methylene group, $47.9 \%$ for the $\gamma$-methylene group and only $7.8 \%$ for the $\beta$-methylene group as presented in Figure 1c. The total deuteration level as estimated by ${ }^{1} \mathrm{H}-\mathrm{NMR}$ was $38.2 \%$. Consequently, the estimated SLD of the d-wax sample is $2.2 \times 10^{-6} \AA^{-2}$. The detailed estimation method and analysis were illustrated in Figure S1 and Table S1.

\subsection{Morphologies of natural wax and reconstituted wax films}

SEM imaging was first carried out from the natural wax morphology present on the adaxial surface of a 3-weeks-old wheat leaf as well as the reconstituted wax films on OTS and Si substrate surfaces, with representative images shown in Figure 2 a, b and Figure S2, respectively. Both natural and reconstituted wax films consist of 2 distinct layers: an 
underlying wax layer and an outer layer of wax protrusions, consistent with our previous observations [22]. Whilst the protrusions present on the reconstituted wax films on Si and OTS surfaces have similar size and density, the distribution of the wax protrusions on the natural leaf surface appears denser. In addition, the sizes of surface protrusions on both natural and reconstituted wax films appear uniform across the scanned sites, though the protrusions on the reconstituted wax films are wider. These observations indicate that the chemical nature and hydrophobicity of the substrates do not have a significant impact on the basic morphological features of the wax films. Thus, the broad similarity to the natural leaf wax films makes the reconstituted ones suitable models for further investigations.

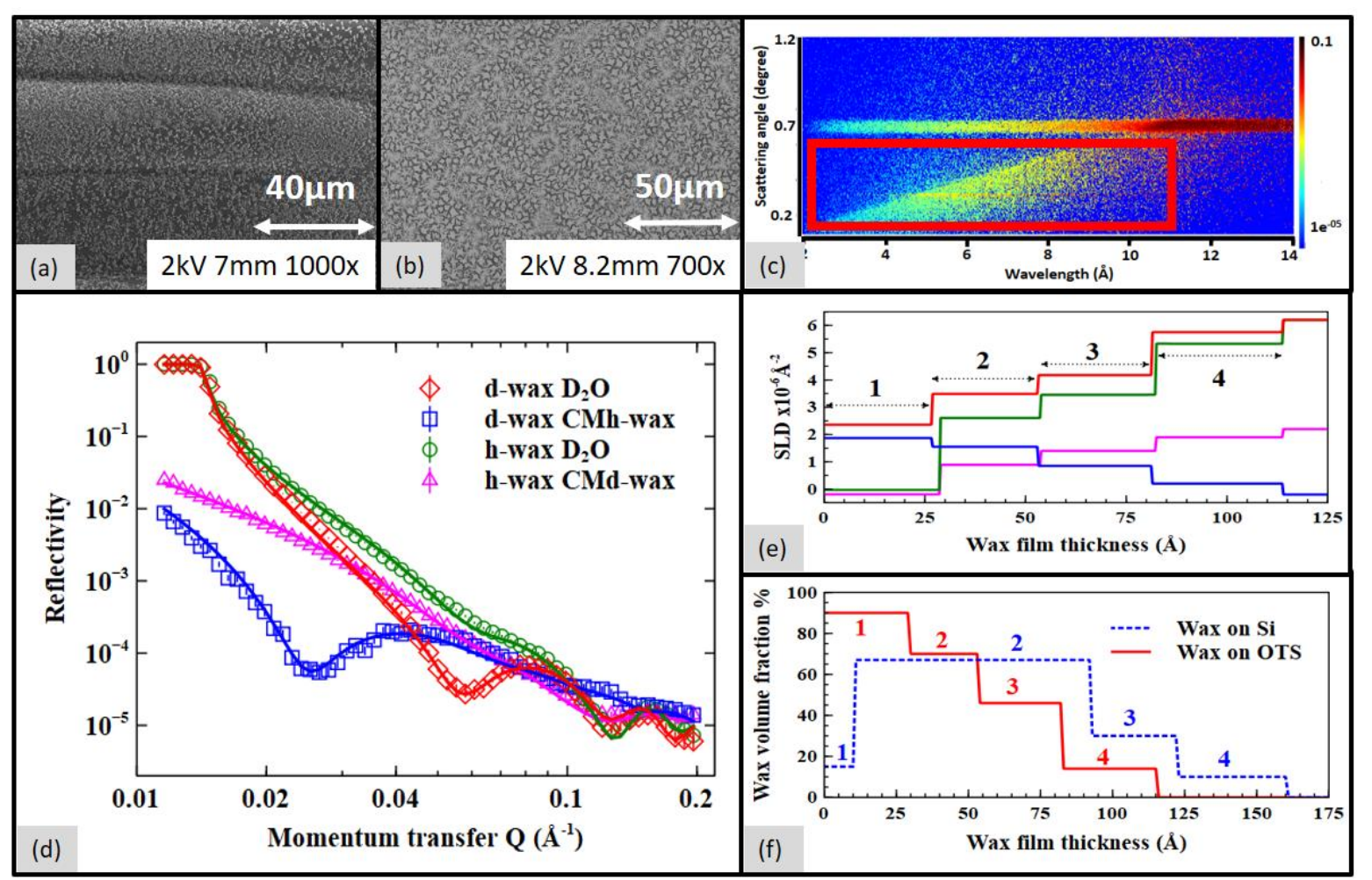

Figure 2: SEM images for the natural wax on the adaxial surface of a 3-weeks-old wheat leaf (a) and from the reconstituted wax film on OTS surface (b); Off-specular and specular neutron reflection measured for the h-wax film on OTS surface in $\mathrm{D}_{2} \mathrm{O}$ (c), off-specular neutron reflection marked by the red rectangular box confirms the presence of surface protrusions; (d) the specular NR profiles measured from wax films reconstituted on the OTS surface, the reflectivity of wax film in $\mathrm{D}_{2} \mathrm{O}$ was rescaled to 1 using the critical egde and the reflectivity for other contrasts was rescaled by multiplying the same factor; (e): corresponding wax film SLDs against film thickness on the OTS surface, 1-4 denotes the four layers fitted; (f): the wax volume fraction profiles against wax film thickness for wax films on $\mathrm{SiO}_{2}$ and OTS surfaces.

\subsection{Structures of reconstituted wax films on OTS and $\mathrm{SiO}_{2}$}


NR was first used to characterise the structure of the native silicon oxide layer. The measured reflectivity profile could be fitted to a uniform layer with a thickness of $15 \pm 2 \AA$ and an SLD of $3.4 \times 10^{-6} \AA^{-2}$, indicating no defects or voids present. The variation of $\pm 2 \AA$ in this case indicated the range of thickness found from different silicon block surfaces.

After surface coating of the OTS monolayer onto silicon block, NR measurements were undertaken at the solid/ $\mathrm{D}_{2} \mathrm{O}$ interface to characterise its structure. A uniform layer was found to be adequate to fit the NR profile, with the structure of the $\mathrm{SiO}_{2}$ layer being taken to be the same as before the OTS layer coating. The thickness of the OTS layer was found to be $23 \pm 2$ $\AA$ with an SLD corresponding to the $80 \%$ OTS coverage. This indicates that the alkyl chains were nearly perpendicular to the surface and that the OTS layer was rather well packed. Further information on the OTS characterisation is given in Figure S3 and Table S2.

Both d-wax and h-wax films reconstituted on the OTS surface were characterised by NR at the solid/liquid interface by simultaneously fitting the reflectivity profiles measured in 4 different contrasts: h-wax in $\mathrm{D}_{2} \mathrm{O}$, h-wax under CMd-wax, d-wax in $\mathrm{D}_{2} \mathrm{O}$ and d-wax under CMh-wax. As shown in Figure 2b, the reconstituted wax films are quite rough due to the appearance of outer protrusions, resulting in strong off-specular reflection. Figure 2c shows both off-specular and specular reflection signals from the reconstituted h-wax film on OTS surface in $\mathrm{D}_{2} \mathrm{O}$ at an incident angle of $0.7^{\circ}$, with off-specular signal highlighted by the red rectangular box.

The measured specular NR profiles (rescaled) with the best fits are shown in Figure 2(d). A 4-layer wax model with each layer containing wax and water in different ratios was found to model the wax films well. This was the minimal number of layers that could simultaneously fit the measured NR profiles in the 4 contrasts. The corresponding SLD profiles for the averaged wax film against film thickness are shown in Figure 2(e). The large variations in SLD indicate the changes in H/D substitution in wax and solvent.

Wax films were also coated onto the bare $\mathrm{SiO}_{2}$ surface and measured by NR under the same film coating and isotopic contrasts. Fitting to the NR profiles measured at the hydrophilic silicon oxide/water interface was also done in the procedure similar to that described earlier and the detailed fitting parameters for wax films on both $\mathrm{SiO}_{2}$ and OTS surfaces are compared in Figure S4 and Tables S3 and S4.

As the neutron reflectivity shown in Figure 2(e) is from specular reflection, it only reveals the structural information of the underlying wax film and the bulk of the protrusions in the 
direction perpendicular to the surface. No clear boundary exists between the underlying wax layer and the outer protrusions region, but the SEM imaging indicates that the inner layer is rather uniform and dense whilst the outer protrusions are comprised of large wax crystals and are thick but low in volume fraction.

The distribution profiles obtained from fitting to the reflectivity profiles measured from the reconstituted wax films on both $\mathrm{SiO}_{2}$ and OTS substrates are shown in Figure 2(f) in the form of volume fraction plotted against thickness, with the actual values obtained from the best 4layer model fits listed in Table 2. Although these wax films show similar surface coverage (the amount of waxes in a given surface area), their structural distributions differ quite obviously. For wax films reconstituted on silica, there is a thin transitional layer between the silicon oxide layer and the dense wax layer with a thickness of $10 \AA$ and a wax volume fraction of 0.15 (layer 1). The low density transition layer must arise from the very different amphiphilic nature between waxes and the $\mathrm{SiO}_{2}$ surface. The main wax layer is roughly $90 \AA$ with a volume fraction of 0.67 (layer 2). The outer region of the protrusions is about $60 \AA$ (layer 3 and 4) and was fitted into 2 further layers to account for changing wax volume fraction. This indicates that the volume fraction of the protrusions decreases with increasing film thickness.

For wax films reconstituted on the OTS layer surface, however, no transition layer was detected. Instead, there was just a dense wax layer with a thickness of roughly $25 \AA$ on the OTS layer and a wax volume fraction of 0.90 (layer 1), followed by the second wax layer of similar thickness but with a lower wax volume fraction of 0.70 (layer 2). Subsequent layers followed similar thicknesses but with decreasing wax volume fraction, indicating increasing defects in the wax film away from the substrate surface, as evident from SEM measurements. The bulk part of the protrusions is also 60-70 $\AA$ and can again be divided into 2 further wax crystal layers (layer 3 and 4) to account for decreasing volume fraction.

The entire wax films on the OTS surface are thinner than those coated on the $\mathrm{SiO}_{2}$ surface but clearly denser. The distributions for protrusions on both types of wax films are however similar. This confirms that the substrate hydrophobicity plays an important role in determining the inner wax film structure but has less significant impact on its outer morphological features. The surface hydrophobicity clearly attracts the assembly of the hydrocarbon tails of the long chain alcohols, resulting in the densest molecular packing in the inner wax layer, with subsequent self-assembly of the long chain alcohol layers retaining 
similar thickness but with increasing defects. In contrast, the hydrophilic silica surface prefers the direct contacts with the hydroxyl groups on the wax molecules, but because the hydroxyl groups are small their alignment to the silica surface is weak against the strong hydrophobic interaction between the long alkyl chains, resulting in the strongly hydrated inner layer of 10 $\AA$. This was followed by the dominant hydrophobic interaction in the self-assembly of the long alcohols forming a rather uniform wax region of some $80 \AA$, but its intermediate volume fraction of 0.64 indicates the existence of defects associated with hydration.

These observations suggest that the long chain alcohols might initiate the self-assembly by forming a molecular monolayer with their initial orientation manifested by the substrate surface, that is, the alkyl chains facing the OTS surface versus the hydroxyl groups facing the silica surface [29]. Because of the opposite orientation of the initiating monolayer, the subsequent templating of the self-assembled structures follows different orders, resulting in different alignment of the bilayers. On the OTS surface, the inner monolayer induces the selfassembly of independent bilayers whilst on the silica surface the inner monolayer initiates a complementary outer monolayer and the process then replicates itself. In all cases, however, the amphiphilicity of the long chain alcohol molecules provides the overall driving force in which the hydrophobic interactions amongst the alkyl chains are dominant and the hydrophilic effects amongst the hydroxyl groups are weak. The imbalance causes the propagation of the defects and limits the number of bilayer formed. On the OTS surface, the NR data led to the resolution of about 4 self-assembled wax monolayers. On the basis of the overall dimension for the main wax film region formed on the silica surface, there could be 2 bilayers aligned on top of each other with some inter-mixing between the alkyl chains. Again, the formation of outer waxy pillars is consistent with the declining packing density inherent of the imbalanced amphiphilic nature of the long chain alcohols.

\begin{tabular}{ccccccccc}
\hline \multirow{2}{*}{ Surfaces } & \multicolumn{2}{c}{ Layer 1 } & \multicolumn{2}{c}{ Layer 2 } & \multicolumn{2}{c}{ Layer 3 } & \multicolumn{2}{c}{ Layer 4 } \\
\cline { 2 - 9 } & $\begin{array}{c}\text { Thickness } \\
/ \AA\end{array}$ & $\begin{array}{c}\text { Wax } \\
\text { fraction }\end{array}$ & $\begin{array}{c}\text { Thickness } \\
/ \AA\end{array}$ & $\begin{array}{c}\text { Wax } \\
\text { fraction }\end{array}$ & $\begin{array}{c}\text { Thickness } \\
/ \AA\end{array}$ & $\begin{array}{c}\text { Wax } \\
\text { fraction }\end{array}$ & $\begin{array}{c}\text { Thickness } \\
/ \AA\end{array}$ & $\begin{array}{c}\text { Wax } \\
\text { fraction }\end{array}$ \\
\hline $\mathbf{S i O}_{2}$ & $10 \pm 1$ & $0.15 \pm 0.01$ & $82 \pm 3$ & $0.67 \pm 0.03$ & $32 \pm 3$ & $0.30 \pm 0.03$ & $38 \pm 4$ & $0.10 \pm 0.02$ \\
\hline OTS & $27 \pm 3$ & $0.90 \pm 0.02$ & $26 \pm 3$ & $0.70 \pm 0.04$ & $30 \pm 2$ & $0.45 \pm 0.03$ & $33 \pm 3$ & $0.14 \pm 0.02$ \\
\hline
\end{tabular}

Table 2: The best-fitted parameters for wax films reconstituted on silica and the OTS surface. It was found that the 4-layer model was about the smallest number of layers required to prodcue the best fits.

Although the main features of the wax films reconstituted onto hydrophilic silica (the underlying wax film and outer wax crystals) remain the same as in our previous study [23], the detailed structure may differ due to the use of different data analysis approaches. In our 
previous NR analysis [22], the Hughes model, consisting of an underlying layer and an outer decaying function, was adopted to treat the wax films. Whilst the Hughes model was merited with few parameters in model fitting it was incapable of predicting the more detailed structural features such as the low density inner layer and the more precise outer layer distribution. Furthermore, the multilayer model adopted here avoids the use of roughness as necessitated in the Hughes model due to the huge difference in volume fraction between the 2 regions [30].

\subsection{Interactions of wax films, surfactants and pesticides}

\subsubsection{SE measurements of binding of $\mathrm{C}_{12} \mathrm{E}_{6}$ and $\mathrm{CP}$ to the reconstituted wax films}

The dynamic change of the wax film when exposed to surfactant and CP was first studied at the solid/liquid interface by SE. Initial measurements were made on wax films coated on the hydrophilic silica. It was observed that the coated wax film was very stable under water and that addition of $\mathrm{C}_{12} \mathrm{E}_{6}$ led to the increase in the total surface materials, indicating the adsorption of $\mathrm{C}_{12} \mathrm{E}_{6}$ onto the coated wax film. As the concentration of $\mathrm{C}_{12} \mathrm{E}_{6}$ went above the $\mathrm{CMC}$, a high proportion of the wax film was removed. The instability of the wax films coated on the hydrophilic $\mathrm{SiO}_{2}$ surface was consistent with what was observed previously [23]. The data shown in Figure 3 describes the interactions between wax films coated on the OTS surface and $\mathrm{C}_{12} \mathrm{E}_{6} / \mathrm{CP}$ at the solid/water interface. It was found that below the $\mathrm{CMC}$ and to the CMC the surfactant also adsorbed onto the wax film as in the case of the adsorption onto wax films coated on the $\mathrm{SiO}_{2}$ surface. Thus, the data shown in Figure 3 focuses on assessing stability above the CMC.

To reflect the main structural feature of the coated wax film in the SE data analysis, a 3-layer model, consisting of silicon oxide layer, the OTS layer and the wax Cauchy layer (A: 1.45, B: 0.01), was used to account for the material changes from the substrate to the solid/liquid interface. Because SE has little structural sensitivity to the exact distribution of the wax nanofilm, it can hardly detect structural difference between the inner underlying layer and the outer crystal layer. Thus, the single wax layer is sufficient to represent the entire wax film. However, SE is highly sensitive to mass changes and the model can help determine the net mass changes of the interfacial film before, during and after its interaction with surfactant and pesticide. Lack of structural sensitivity also means that SE could not distinguish between wax and the bound surfactant, but the 3-layer model helped to derive the amount of $\mathrm{C}_{12} \mathrm{E}_{6}$ and $\mathrm{CP}$ binding from changes in the total mass of the wax film during and after the interactions. Figure 3 depicts the typical mass changes associated with the binding of $\mathrm{C}_{12} \mathrm{E}_{6}$ and $\mathrm{CP}$ onto 
wax films reconstituted onto the OTS surface, with the measurement starting in pure water defining the stability of the wax film itself and its amount quantified in $\mathrm{ng} / \mathrm{mm}^{2}$. The initial mass of the wax film was $3.8 \mathrm{ng} / \mathrm{mm}^{2}$.

A common feature of all the measurements shown in Figure 3 is the fast adsorption and desorption under different conditions, similar to the behaviour as observed below the CMC on wax films coated on both model substrates. At $0.3 \mathrm{CMC}$ and $1 \mathrm{CMC}$, the total amount of surface mass increased to $4.05 \mathrm{ng} / \mathrm{mm}^{2}$ and $4.3 \mathrm{ng} / \mathrm{mm}^{2}$, respectively, indicating $\mathrm{C}_{12} \mathrm{E}_{6}$ adsorption of $0.25 \mathrm{ng} / \mathrm{mm}^{2}$ and $0.5 \mathrm{ng} / \mathrm{mm}^{2}$. Water rinsing did not lead to any mass reduction, indicating the wax film was rather stable when exposed to surfactants well below and close to CMC. At 10CMC, the total amount of the surface mass increased to $4.4 \mathrm{ng} / \mathrm{mm}^{2}$. Water rinsing led to the lowering of the total mass to even below the mass of the wax film, 3.6 $\mathrm{ng} / \mathrm{mm}^{2}$. This confirmed partial wax removal and indicated that $\mathrm{C}_{12} \mathrm{E}_{6}$ adsorption amount was $0.8 \mathrm{ng} / \mathrm{mm}^{2}$. In fact, the baseline as shown in Figure 3 shows a steady decrease over the course of the experiments, signifying the progressive removal of some of the wax films deposited. Increase in surfactant concentration to 20CMC led to almost the same amount of surfactant adsorption. Dissolution of $\mathrm{CP}$ into $\mathrm{C}_{12} \mathrm{E}_{6}$ micelles allowed the measurement of the co-adsorption achieving the highest adsorbed amount of $0.9 \mathrm{ng} / \mathrm{mm}^{2}$. The difference of 0.10 $0.15 \mathrm{ng} / \mathrm{mm}^{2}$ indicates the contribution from CP under these conditions, but rinsing helps remove them completely. In spite of the large errors, the mass ratio of $\mathrm{CP}$ to $\mathrm{C}_{12} \mathrm{E}_{6}$ as observed here is close to the ratio of 1:8 as observed in the bulk solution. These experiments show high stability of the wax films coated on OTS surface, in spite of small but noticeable removal of the waxes as experiments progressed.

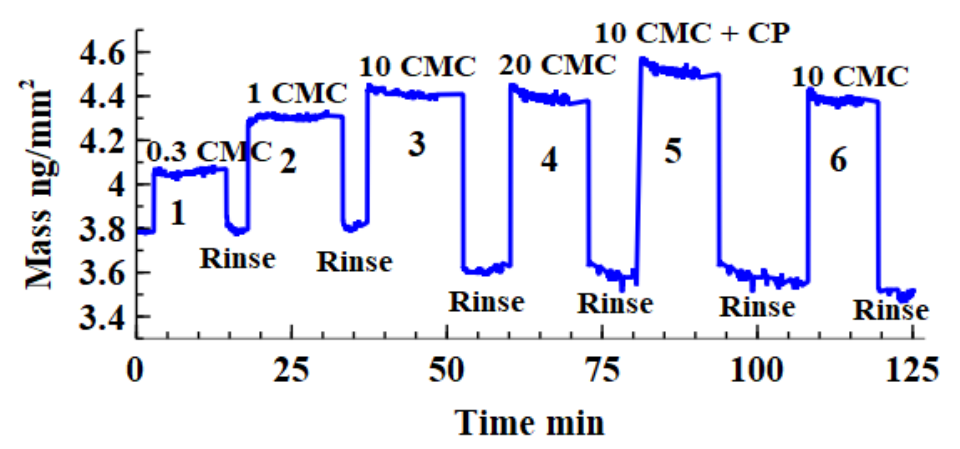

Figure 3: Mass changes from the wax films reconstituted onto the OTS surface when exposed to $\mathrm{C}_{12} \mathrm{E}_{6}$ at $10 \mathrm{CMC}, 20 \mathrm{CMC}$, with $\mathrm{CP}$ at $0.3 \mathrm{CMC}, 1 \mathrm{CMC}, 10 \mathrm{CMC}$ and $10 \mathrm{CMC}$ marked by 1-6, with pure water rinses following each measurement. 


\subsubsection{NR measurements of interaction between wax film on the OTS surface with $\mathrm{C}_{12} \mathrm{E}_{6}$ and $\mathrm{CP}$}

Following the SE measurements as shown in Figure 3, the NR measurements was first undertaken at $10 \mathrm{CMC}$ under 5 isotopic contrasts: h-wax with $\mathrm{hC}_{12} \mathrm{hE}_{6}$ in $\mathrm{D}_{2} \mathrm{O}$, h-wax with $\mathrm{dC}_{12} \mathrm{hE}_{6}$ in $\mathrm{D}_{2} \mathrm{O}$, h-wax with $\mathrm{dC}_{12} \mathrm{hE}_{6}$ in CMh-wax, d-wax with $\mathrm{hC}_{12} \mathrm{hE}_{6}$ in $\mathrm{D}_{2} \mathrm{O}$ and d-wax with $\mathrm{hC}_{12} \mathrm{hE}_{6}$ in $\mathrm{CMd}$-wax, with $\mathrm{NR}$ profiles shown in Figure $4 \mathrm{a}$ and the best fitted parameters in Table S5.

Comparing Figure $4 \mathrm{a}$ with Figure $2 \mathrm{~d}$, the NR profiles for $\mathrm{h} / \mathrm{d}$-waxes in $\mathrm{D}_{2} \mathrm{O}$ changed with the presence of surfactants. The remaining contrasts highlight the distribution of the $\mathrm{C}_{12}$ tail within the wax film (h-wax with $\mathrm{dC}_{12} \mathrm{hE}_{6}$ in $\mathrm{CMh}$-wax) as well as the whole surfactant distribution (d-wax with $\mathrm{hC}_{12} \mathrm{hE}_{6}$ in $\mathrm{CMd}-\mathrm{wax}$ ). As these 2 contrasts have a nonlinear region in reflectivity profiles presented on a logarithmic reflectivity scale against LogQ shown in Figure $4 \mathrm{a}$, their combined fittings could lead to the determination of the amount of surfactant molecules across the wax films. The minimum SLD difference that can be effectively determined in the data analysis is $\pm 0.1 \times 10^{-6} \AA^{-2}$, equivalent to about $2 \%$ of the surfactant in the film $[31,32]$.

As SE measurements have revealed that the wax film was stable when exposed to surfactants, the film thickness was considered unchangeable. As explained previously, the structures of the silicon oxide and OTS layers were fixed at $15 \AA$ and $23 \AA$ in NR data analysis. It was necessary to divide the base wax film into 3 layers to account for structural inhomogeneity arising from surfactant penetration. The thickness for each layer (from the wax/OTS interface to the dense/sparse wax interface) was $12 \AA, 13 \AA$ and $25 \AA$, respectively. The division of these layers helped take into account of the volume of the head group of $\mathrm{C}_{12} \mathrm{E}_{6}$ which is slightly larger than that of its tail (volume ratio:1.08:1) [13]. In comparison, the wax protrusion layer was almost evenly divided into 4 layers to handle changes in wax volume fraction. The thickness for each layer was $15 \AA$.

The reflectivity profiles were fitted to a 7-layer wax model. The SLD profiles generated are shown in Figure $4 \mathrm{~b}$. The volume fractions for the wax film and surfactant constituents are shown against distance from the OTS surface in Figure 4c. Comparing Figure 4c to Figure 2f, the wax volume fractions decrease slightly due to their mild interaction with the surfactant, but the main feature remains the same. The surfactant chains aggregate within the wax film at distances of 0-12 $\AA$ and 50-115 $\AA$ from the OTS surface. As the ethoxylate heads bind to the 
alkyl tails, the heads must be distributed in the same or adjacent layer. Taking into account of the contributions from the other contrasts, we found that the head groups mainly distributed in the regions of 12-25 $\AA$ and 50-115 $\AA$. The base layer of the dense wax is $50 \AA$ and the surfactants have either penetrated to the wax surface closest to the OTS layer or adsorb to the wax protrusion or the surface of the dense wax layer. There is a gap with a negligible amount of surfactant in the wax film at 25-50 $\AA$. Whilst the reason for this is unclear, a possible explanation is the different molecular packing between inner and outer wax layers. The inner layer of the wax film is comprised of a 2-dimenisonal layers as in the case of a LangmuirBlodgett film [29] while the outer protrusions contain the three dimensional crystals. The gap is a transition between the underlying wax film and the top protrusions where the chemical composition and physical morphology are unfavourable for surfactants to bind.

The alignment of wax molecules in the inner layer is confirmed by the orientation of surfactants. In analysing the surfactant distribution within the inner denser region, there was clear indication that surfactant tails were oriented towards the OTS surface with their heads pointed towards the wax film; the tail layer was $12 \AA$ thick and the head layer was $13 \AA$ thick, providing a thickness of $25 \AA$ for the total layer. In the outer region of $50-115 \AA$, the surfactant distribution did not bear any orientation due to the larger space span, but the total volume ratio in this area for the heads and tails was fixed at 1.08. This suggested that the surfactants form surface micellar aggregates on the wax film surface and protrusions, consistent with our previous observations [23].

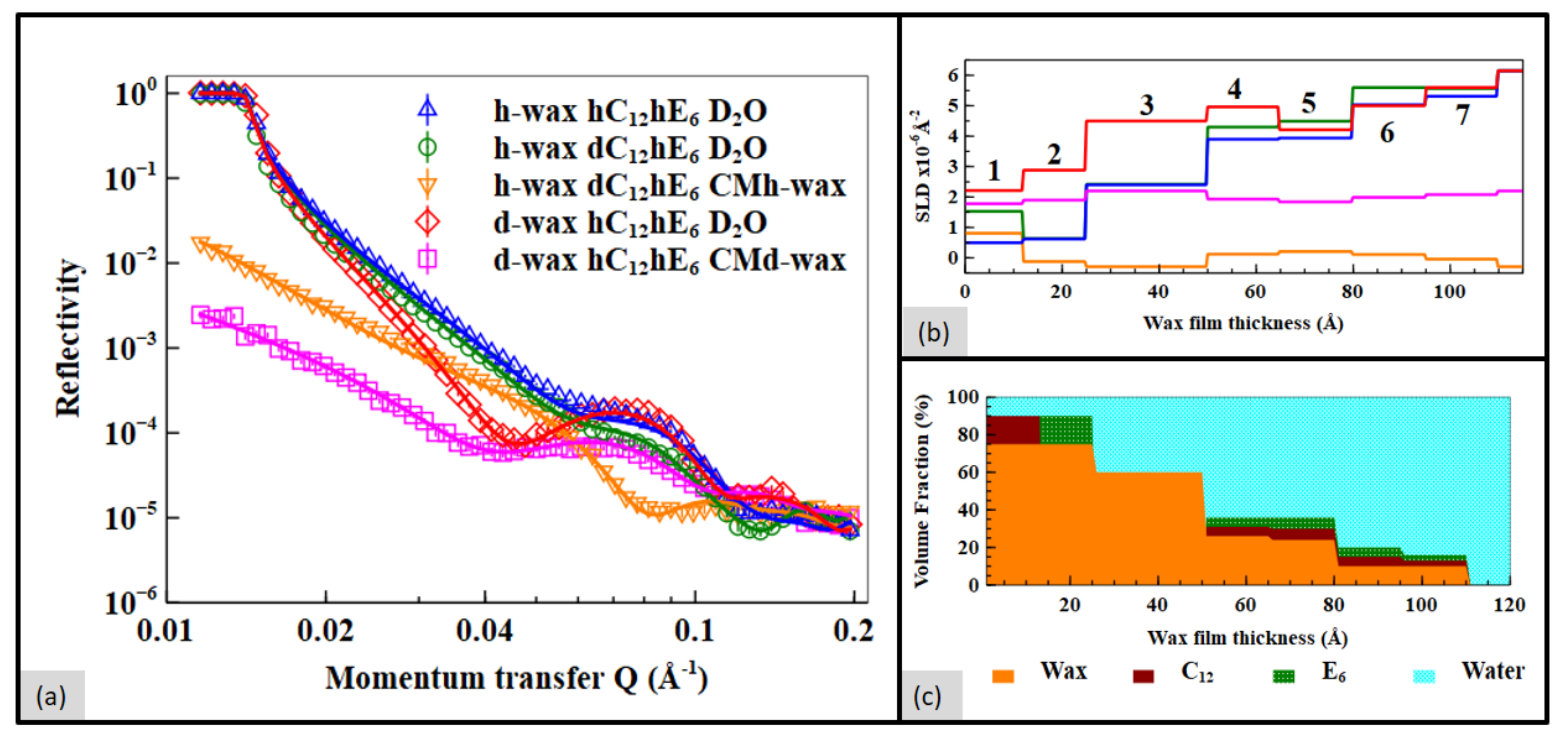

Figure 4: (a): the specular NR profiles for wax films on the OTS surface interacting with $\mathrm{C}_{12} \mathrm{E}_{6}$ at $10 \mathrm{CMC}$ in 5 isotopic contrasts; the reflectivity for $\mathrm{D}_{2} \mathrm{O}$ runs was rescaled to 1 and 
the reflectivity for other contrasts was rescaled by multiplying the same factor; (b) SLD profiles against wax film thickness for wax films on the OTS surface interacting with $\mathrm{C}_{12} \mathrm{E}_{6}$ at $10 \mathrm{CMC}$ in 5 isotropic contrasts; (c) volume fraction profiles for wax, surfactant tail and head, and water against distance from the OTS surface for wax films on the OTS surface interacting with $\mathrm{C}_{12} \mathrm{E}_{6}$ at $10 \mathrm{CMC}$.

To locate pesticide in the wax film, the measurements on wax- $\mathrm{C}_{12} \mathrm{E}_{6}-\mathrm{CP}$ interactions were then performed in 4 contrasts: h-wax with $\mathrm{hC}_{12} \mathrm{hE}_{6}$ and $\mathrm{CP}$ in $\mathrm{D}_{2} \mathrm{O}$, h-wax with $\mathrm{dC}_{12} \mathrm{hE}_{6}$ and $\mathrm{CP}$ in $\mathrm{D}_{2} \mathrm{O}$, h-wax with $\mathrm{dC}_{12} \mathrm{hE}_{6}$ and $\mathrm{CP}$ in $\mathrm{CMh}$-wax and h-wax with $\mathrm{hC}_{12} \mathrm{hE}_{6}$ and $\mathrm{CP}$ in CMH-wax, with the NR profiles shown in Figure 5 and the best fitting parameters in Table S6. Among these 4 contrasts, the h-wax with $\mathrm{hC}_{12} \mathrm{hE}_{6}$ and $\mathrm{CP}$ in $\mathrm{CMh}$-wax was used to highlight the distribution of CP. As reflectivity (presented on a logarithmic scale) is almost linearly proportional to $\log \mathrm{Q}$, there is no local accumulation of $\mathrm{CP}$ in specific layers across the whole wax film with volume fraction over $10 \%$ because this would be resolved from the SLD distribution with confidence. Also, comparing Figure 5 with Figure $4 \mathrm{a}$, there is little observable difference in the other 3 contrasts, indicating that the solubilised pesticide has insignificant impact on nonionic surfactant behaviour in the wax films.

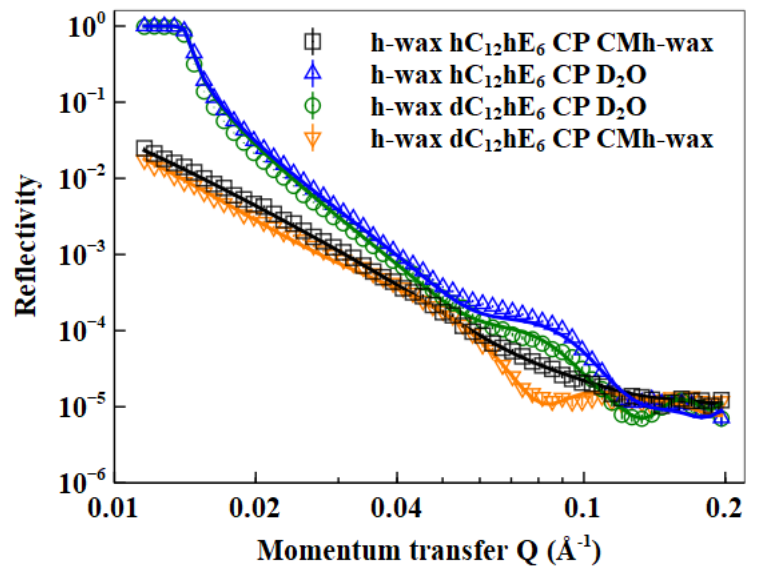

Figure 5: Specular NR profiles from the wax films coated on the OTS surface interacting with $\mathrm{C}_{12} \mathrm{E}_{6}$ solubilised with $\mathrm{CP}$ at $10 \mathrm{CMC}$ in 4 contrasts; the reflectivity profiles in $\mathrm{D}_{2} \mathrm{O}$ were rescaled to 1 and the reflectivity profiles in other contrasts were rescaled by multiplying the same factor.

Neutron reflection thus revealed that $\mathrm{CP}$ was distributed across the entire wax film, as it neither gathered in a specific layer nor affected the nonionic surfactant distribution in any particular manner. Given the limited resolution in the interfacial wax films from NR, it is difficult to extrapolate how $\mathrm{CP}$ molecules were incorporated into wax films, but it is likely 
that they were facilitated by surfactant micelles that become adopted into the wax film environment. It should be noted from previous surfactant solution studies that although CP solubilisation dehydrates the micellar shell and increases the micellar hydrophobicity [13], further wax solubilisation into the micelles re-equilibrates the system, releases some $\mathrm{CP}$ and decreases the micellar hydrophobicity [14]. Therefore, it is unsurprising to see that the adsorption of nonionic surfactant molecules has not altered significantly in the presence of $\mathrm{CP}$, i.e., the nonionic surfactant molecules still form surface micelles in similar sizes on the wax film with $\mathrm{CP}$ pre-solubilisation. In addition to the penetration into the wax film, some pesticides should also stay with the surfactants in the protrusion layer as surfactants are also adsorbed on the wax protrusions.

Figure 6 offers schematic depiction of how $\mathrm{C}_{12} \mathrm{E}_{6}$ and $\mathrm{CP}$ molecules interact with the wax film reconstituted on the OTS surface. Both surfactant and pesticide molecules adsorb onto the wax protrusions and can also penetrate into the underlying wax film. Surfactant micelles can remove solubilised wax molecules from the film, a removal process that agrees well with previous observations [12, 23].

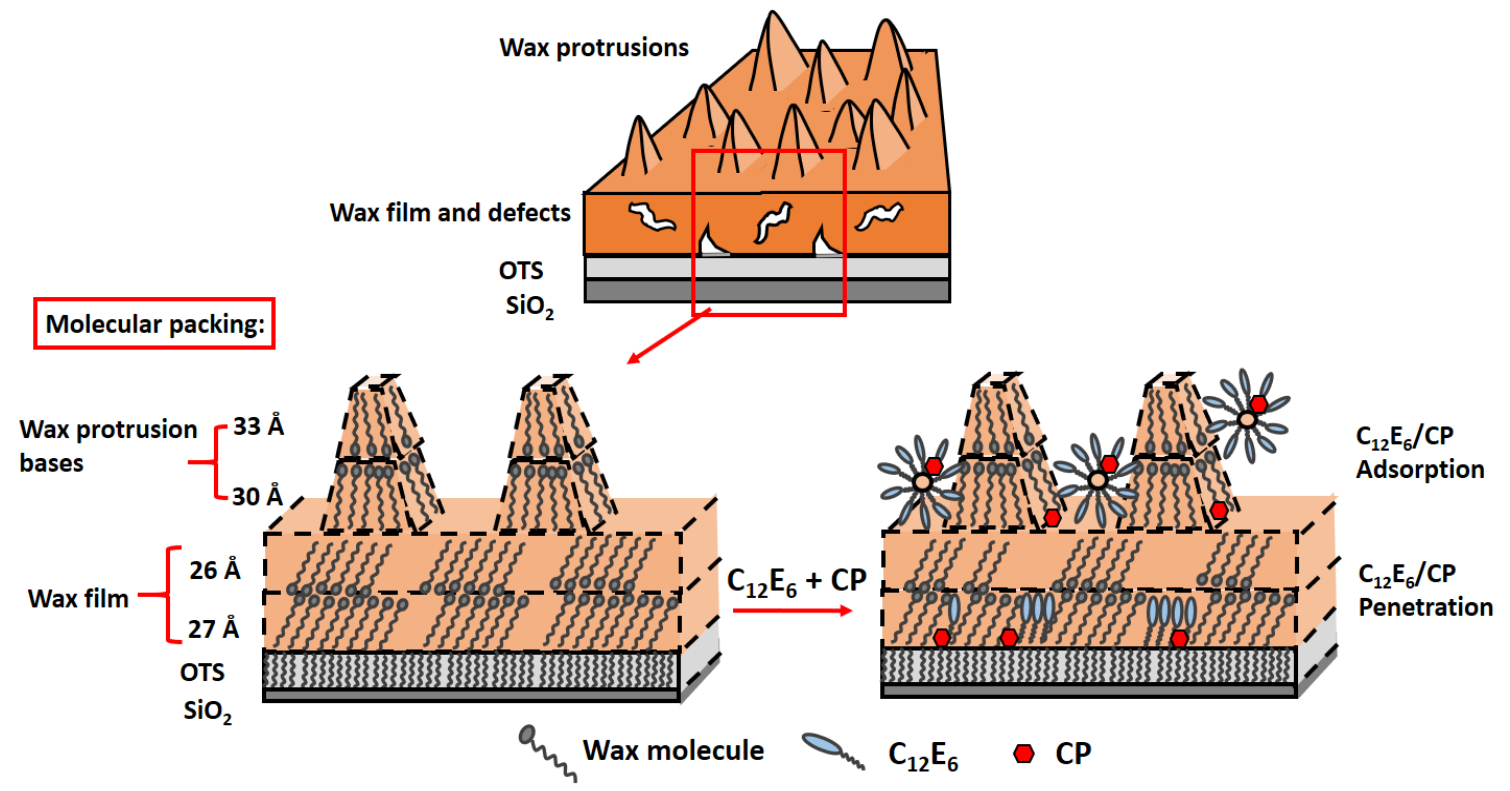

Figure 6: Schematic sketches of the wax- $\mathrm{C}_{12} \mathrm{E}_{6}-\mathrm{CP}$ interaction process leading to dissolution and removal of waxes by surfactant micelles at the molecular level and association of surfactant and CP molecules into the inner interfacial region of the wax film and onto the wax protrusions.

\section{Conclusions}


This work has shown that wheat wax films reconstituted on both hydrophilic silica and hydrophobic OTS surfaces bear similar morphological features, that is, the underlying wax film and outer wax protrusions. The 2-layer characteristics also bear close resemblance to the morphological features from the native wheat wax films [21, 22, 33-35]. The outer wax surface crystals exhibit similar features when reconstituted on both hydrophilic and hydrophobic surfaces but the actual thickness and volume fraction distribution across each type of wax films are different. This results in very different wax film stability when treated with the nonionic surfactant $\mathrm{C}_{12} \mathrm{E}_{6}$, suggesting that the hydrophilicity of the substrate has a significant impact on the wax film stability. The wax films reconstituted onto the hydrophobic surface are more stable and robust when exposed to $\mathrm{C}_{12} \mathrm{E}_{6}$. The OTS layer has a higher binding affinity with the wax films, resulting in a denser base layer and more robust wax films.

The impact of exposure of the wax films reconstituted onto the OTS surface to $\mathrm{C}_{12} \mathrm{E}_{6}$ and $\mathrm{CP}$ was studied by SE and NR above the CMC to mimic the practical scenario of agri-sprays in which nonionic surfactant concentrations are usually well above their CMCs. The overall structure of the wax film remained stable under $\mathrm{C}_{12} \mathrm{E}_{6}$ exposure even at $20 \mathrm{CMC}$. The results indicated that the surfactant molecules are not only able to adsorb onto outer wax protrusions but also penetrate into the underlying wax film. The pesticide molecules are able to adsorb onto wax films when delivered by nonionic surfactant micelles. The penetration of surfactant molecules then gives clear pathways for pesticides to diffuse into wax films. As different plant species have distinct wax film morphologies, future work will examine how pesticide and surfactant interact with differed wax films reconstituted from various plant species.

\section{Acknowledgements}

We thank Syngenta for funding this work. We also thank the University of Manchester for a studentship to XH, EP, HG, ML, PH, HL and ISIS Neutron Facility at Rutherford Appleton Laboratory, STFC for neutron beam times on SURF and Offspec. HG and ML thank the research funding provided by the Chinese Scholarship Council.

\section{Supporting Information}

Further data in the form of ${ }^{1} \mathrm{H}-\mathrm{NMR}$ spectra measured from protonated and deuterated waxes, interpretation of SE and NR data analysis, SEM image for reconstituted wax film on hydrophilic substrate, NR profiles for bare OTS substrate, wax film on hydrophilic surface, 
wax films on hydrophobic surface after surfactant binding are shown in the Supporting Information document. Supporting data are available on request from the corresponding author.

\section{References}

[1] W. Aktar, D. Sengupta, A. Chowdhury, Impact of pesticides use in agriculture: their benefits and hazards, Interdisciplinary Toxicology 2(1) (2009) 1-12.

[2] M. Kah, R.S. Kookana, A. Gogos, T.D. Bucheli, A critical evaluation of nanopesticides and nanofertilizers against their conventional analogues, Nature Nanotechnology 13(8) (2018) 677.

[3] D.P. Sachdev, S.S. Cameotra, Biosurfactants in agriculture, Applied Microbiology and Biotechnology 97(3) (2013) 1005-1016.

[4] J. Zhou, C. Li, Z. Jiang, L. Wang, H. Su, H. Li, C. Deng, Q. Wang, J. Wang, F. Jin, Occurrences of the typical agricultural nonionic surfactants tristyrylphenol ethoxylates in cherries (Cerasus pseudocerasus), peaches (Amygdalus persica), and kiwifruit (Actinidia Chinensis) and the implications of human exposure in China, Journal of Agricultural and Food Chemistry 67(10) (2019) 2999-3005.

[5] L. Wang, X. Li, G. Zhang, J. Dong, J. Eastoe, Oil-in-water nanoemulsions for pesticide formulations, Journal of Colloid and Interface Science 314(1) (2007) 230-235.

[6] D. Seaman, Trends in the formulation of pesticides - an overview, Pesticide Science 29(4) (1990) 437-449.

[7] L. Zheng, C. Cao, L. Cao, Z. Chen, Q. Huang, B. Song, Bounce Behavior and Regulation of Pesticide Solution Droplets on Rice Leaf Surfaces, Journal of Agricultural and Food Chemistry 66(44) (2018) 11560-11568.

[8] M. Song, D. Hu, X. Zheng, L. Wang, Z. Yu, W. An, R. Na, C. Li, N. Li, Z. Lu, Enhancing droplet deposition on wired and curved superhydrophobic leaves, ACS Nano 13(7) (2019) 7966-7974.

[9] M. Riederer, M. Burghardt, S. Mayer, H. Obermeier, J. Schoenherr, Sorption of monodisperse alcohol ethoxylates and their effects on the mobility of 2, 4-D in isolated plant cuticles, Journal of Agricultural and Food Chemistry 43(4) (1995) 1067-1075.

[10] L. Schreiber, J. Schönherr, Analysis of foliar uptake of pesticides in barley leaves: role of epicuticular waxes and compartmentation, Pest Management Science 36(3) (1992) 213-221.

[11] C. Wang, Z. Liu, Foliar uptake of pesticides-present status and future challenge, Pesticide Biochemistry and Physiology 87(1) (2007) 1-8.

[12] A. Fagerström, V. Kocherbitov, T. Ruzgas, P. Westbye, K. Bergström, J. Engblom, Effects of surfactants and thermodynamic activity of model active ingredient on transport over plant leaf cuticle, Colloids and Surfaces B: Biointerfaces 103 (2013) 572-579.

[13] X. Hu, H. Gong, Z. Li, S. Ruane, H. Liu, E. Pambou, C. Bawn, S. King, K. Ma, P. Li, F. Padia, G. Bell, J.R. Lu, What happens when pesticides are solubilized in nonionic surfactant micelles, Journal of Colloid and Interface Science 541 (2019) 175-182.

[14] X. Hu, H. Gong, Z. Li, S. Ruane, H. Liu, P. Hollowell, E. Pambou, C. Bawn, S. King, S. Rogers, K. Ma, P. Li, F. Padia, G. Bell, J.R. Lu, How does solubilisation of plant waxes into nonionic surfactant micelles affect pesticide release?, Journal of Colloid and Interface Science 556 (2019) 650-657.

[15] L. Schreiber, M. Riederer, K. Schorn, Mobilities of organic compounds in reconstituted cuticular wax of barley leaves: effects of monodisperse alcohol ethoxylates on diffusion of pentachlorophenol and tetracosanoic acid, Pesticide Science 48(2) (1996) 117-124.

[16] M. Burghardt, L. Schreiber, M. Riederer, Enhancement of the diffusion of active ingredients in barley leaf cuticular wax by monodisperse alcohol ethoxylates, Journal of Agricultural and Food Chemistry 46(4) (1998) 1593-1602. 
[17] T. Yang, B. Zhao, A.J. Kinchla, J.M. Clark, L. He, Investigation of pesticide penetration and persistence on harvested and live basil leaves using surface-enhanced Raman scattering mapping, Journal of Agricultural and Food Chemistry 65(17) (2017) 3541-3550.

[18] S.O. Duke, Interaction of chemical pesticides and their formulation ingredients with microbes associated with plants and plant pests, Journal of Agricultural and Food Chemistry 66(29) (2018) 7553-7561.

[19] M. Song, Z. Liu, Y. Ma, Z. Dong, Y. Wang, L. Jiang, Reducing the contact time using macro anisotropic superhydrophobic surfaces-Effect of parallel wire spacing on the drop impact, NPG Asia Materials 9(8) (2017) e415.

[20] M. Song, J. Ju, S. Luo, Y. Han, Z. Dong, Y. Wang, Z. Gu, L. Zhang, R. Hao, L. Jiang, Controlling liquid splash on superhydrophobic surfaces by a vesicle surfactant, Science Advances 3(3) (2017) e1602188. [21] K. Koch, W. Barthlott, S. Koch, A. Hommes, K. Wandelt, W. Mamdouh, S. De-Feyter, P. Broekmann, Structural analysis of wheat wax (Triticum aestivum, c.v. 'Naturastar' L.): from the molecular level to three dimensional crystals, Planta 223(2) (2006) 258-270.

[22] E. Pambou, Z. Li, M. Campana, A. Hughes, L. Clifton, P. Gutfreund, J. Foundling, G. Bell, J.R. Lu, Structural features of reconstituted wheat wax films, Journal of The Royal Society Interface 13(120) (2016) 20160396.

[23] E. Pambou, X. Hu, Z. Li, M. Campana, A. Hughes, P. Li, J.R. Webster, G. Bell, J.R. Lu, Structural features of reconstituted cuticular wax films upon interaction with nonionic surfactant C12E6, Langmuir 34(11) (2018) 3395-3404.

[24] J.R. Lu, 2 Neutron reflection studies of interactions between surfactants and proteins at interfaces, Annual Reports Section "C" (Physical Chemistry) 98(0) (2002) 3-32.

[25] F.N. Padia, M. Yaseen, B. Gore, S. Rogers, G. Bell, J.R. Lu, Influence of molecular structure on the size, shape, and nanostructure of nonionic CnEm surfactant micelles, The Journal of Physical Chemistry B 118(1) (2013) 179-188.

[26] J. Wang, D. Jia, K. Tao, C. Wang, X. Zhao, M. Yaseen, H. Xu, G. Que, J.R. Webster, J.R. Lu, Interfacial assembly of lipopeptide surfactants on octyltrimethoxysilane-modified silica surface, Soft Matter 9(40) (2013) 9684-9691.

[27] J. Jin, L. Wang, Z.L. Zheng, J.W. Zhang, X.Z. Hu, J.R. Lu, D. Etor, C. Pearson, A.M. Song, D. Wood, A.J. Gallant, C. Balocco, Metal-insulator-metal diodes based on alkyltrichlorosilane self-assembled monolayers, Aip Adv 9(6) (2019).

[28] A. Nelson, Co-refinement of multiple-contrast neutron/X-ray reflectivity data using MOTOFIT, Journal of Applied Crystallography 39(2) (2006) 273-276.

[29] D.K. Schwartz, Langmuir-Blodgett film structure, Surface Science Reports 27(7-8) (1997) 245-334. [30] J. Lu, T. Su, R. Thomas, J. Penfold, R. Richards, The determination of segment density profiles of polyethylene oxide layers adsorbed at the air-water interface, Polymer 37(1) (1996) 109-114.

[31] D. Ciumac, R.A. Campbell, H. Xu, L.A. Clifton, A.V. Hughes, J.R. Webster, J.R. Lu, Implications of lipid monolayer charge characteristics on their selective interactions with a short antimicrobial peptide, Colloids and Surfaces B: Biointerfaces 150 (2017) 308-316.

[32] D. Ciumac, R.A. Campbell, L.A. Clifton, H. Xu, G. Fragneto, J.R. Lu, Influence of acyl chain saturation on the membrane-binding activity of a short antimicrobial Peptide, ACS Omega 2(11) (2017) 7482-7492.

[33] W. Barthlott, C. Neinhuis, D. Cutler, F. Ditsch, I. Meusel, I. Theisen, H. Wilhelmi, Classification and terminology of plant epicuticular waxes, Botanical Journal of the Linnean Society 126(3) (1998) 237-260.

[34] K. Koch, C. Neinhuis, H.J. Ensikat, W. Barthlott, Self assembly of epicuticular waxes on living plant surfaces imaged by atomic force microscopy (AFM), Journal of Experimental Botany 55(397) (2004) 711-718.

[35] K. Koch, B. Bhushan, W. Barthlott, Diversity of structure, morphology and wetting of plant surfaces, Soft Matter 4(10) (2008) 1943-1963. 